Research Article

\title{
Obesity Potentiates Esophageal Squamous Cell Carcinoma Growth and Invasion by AMPK-YAP Pathway
}

\author{
Jia-Huang Liu $\mathbb{D}^{1,2}$ Qi-Fei Wu $\mathbb{D},{ }^{1}$ Jun-Ke Fu $\mathbb{D},{ }^{1}$ Xiang-Ming Che $\mathbb{D}^{2}$, and Hai-Jun Li $\mathbb{D}^{1}$ \\ ${ }^{1}$ Department of Thoracic Surgery, The First Affiliated Hospital of Xi'an Jiaotong University, Xi'an 710061, China \\ ${ }^{2}$ Department of General Surgery, The First Affiliated Hospital of Xi'an Jiaotong University, Xi'an 710061, China
}

Correspondence should be addressed to Xiang-Ming Che; chexiang@mail.xjtu.edu.cn and Hai-Jun Li; lihaijun0304@163.com

Received 17 May 2020; Revised 1 October 2020; Accepted 30 November 2020; Published 11 December 2020

Academic Editor: Luis Alberto Ponce-Soto

Copyright (C) 2020 Jia-Huang Liu et al. This is an open access article distributed under the Creative Commons Attribution License, which permits unrestricted use, distribution, and reproduction in any medium, provided the original work is properly cited.

\begin{abstract}
Obesity could increase the risk of esophageal squamous cell carcinoma (ESCC) and affect its growth and progression, but the mechanical links are unclear. The objective of the study was to explore the impact of obesity on ESCC growth and progression utilizing in vivo trials and cell experiments in vitro. Diet-induced obese and lean nude mice were inoculated with TE-1 cells, then studied for 4 weeks. Serum glucose, insulin, leptin, and visfatin levels were assayed. Sera of nude mice were obtained and then utilized to culture TE-1. MTT, migration and invasion assays, RT-PCR, and Western blotting were used to analyze endocrine effect of obesity on cell proliferation, migration, invasion, and related genes expression of TE- 1 . Obese nude mice bore larger tumor xenografts than lean animals, and were hyperglycemic and hyperinsulinemic with an elevated level of leptin and visfatin in sera, and also were accompanied by a fatty liver. As for the subcutaneous tumor xenograft model, tumors were more aggressive in obese nude mice than lean animals. Tumor weight correlated positively with mouse body weight, liver weight of mice, serum glucose, HOMA-IR, leptin, and visfatin. Obesity prompted significant TE-1 cell proliferation, migration, and invasion by endocrine mechanisms and impacted target genes. The expression of AMPK and p-AMPK protein decreased significantly $(P<0.05)$; MMP9, total YAP, p-YAP, and nonphosphorylated YAP protein increased significantly $(P<0.05)$ in the cells cultured with conditioned media and xenograft tumor from the obese group; the mRNA expression of AMPK decreased significantly $(P<0.05)$; YAP and MMP9 mRNA expression increased significantly $(P<0.05)$ in the cells exposed to conditioned media from the obese group. In conclusion, the altered adipokine milieu and metabolites in the context of obesity may promote ESCC growth in vivo; affect proliferation, migration, and invasion of ESCC cells in vitro; and regulate MMP9 and AMPK-YAP signaling pathway through complex effects including the endocrine effect.
\end{abstract}

\section{Introduction}

Esophageal carcinoma (EC) is one of the most common gastrointestinal malignancies in the world. ESCC and esophageal adenocarcinoma (EAC) account for more than 95\% of EC. ESCC is more predominant in South America, Africa, and Asia [1] and acts as the leading cause of cancer-related death in China [2]. ESCC has a poor prognosis and high mortality rate, most of the patients are diagnosed in the middle or advanced stage, and the general outcome remains very poor for overall 5-year survival rates of about 10\% [3]. Exploring the mechanisms of ESCC growth and development is therefore critical to identify novel therapeutic targets for desperately needed adjuvant treatment.
Obesity is a worldwide health issue, and its prevalence has doubled since 1980 in more than 70 countries and continuously increased in most countries [4]. Obesity is proved to be a risk factor for developing a variety of malignancies, including EAC, cardia gastric cancer, and breast cancer [5-7]. The relationship between obesity and ESCC is very complicated, and the obesity paradox in cancer that obesity may promote cancer development, but not correlate with overall survival of these patients, exists, especially for ESCC $[5,8]$, and a consensus is very hard to reach. But the mainstream opinion is that obesity, especially abdominal obesity, may be correlated with an increased risk for $\operatorname{ESCC}[9,10]$. Dysregulated metabolism that stems from obesity, including hyperglycemia, insulin resistance, and dyslipidemia, could 
further affect tumor growth and development. Obesity also has cumulative white adipose tissue (WAT). WAT acts as an active endocrine organ and secretes a number of soluble mediators named as adipokines which play a dynamic role in regulating metabolism, inflammation, immunity, and so on; the changed adipokine milieu of obesity also impacts on potentiating the development of diseases such as diabetes, cardiovascular disease, and cancer $[11,12]$. Adipokines such as leptin and other growth factors secreted on the background of obesity may influence cancer cell survival and solid tumor growth [11]. Leptin positively correlates with adipose storage and nutritional state and plays an important role in energy balance and appetite control and is also known as a potential mediator of obesity-associated cancers [13]. The new adipokine, visfatin, also mainly secreted by visceral fat, elevates with the increase of obesity [14] and coordinates with glucose and lipid metabolism. Visfatin may also contribute to being a significant mechanistic link in the network of factors influencing obesity-related tumor growth [15].

Yes-associated protein (YAP), a transcriptional coactivator, functions downstream of the Hippo signaling pathway and regulates multiple cellular processes. Dysregulation of the Hippo-YAP pathway existed frequently in human cancers, including oral squamous cell carcinoma [16], gastric cancer [17], and ESCC [18]. YAP shuttles between the cytoplasm and nucleus, where it interacts with transcription factors, particularly TEA domain (TEAD) family members, then prompts proproliferative and antiapoptotic gene expression [19]. When the upstream Hippo kinase receives an extracellular signal to inhibit cell growth, YAP is phosphorylated and inactivated in the cytoplasm, then gets subsequent ubiquitin-mediated proteasomal degradation. By contrast, when the kinase gets a growth prompting signal, hypophosphorylated YAP translocates into the nucleus and induces the expression of target proproliferative and antiapoptotic genes. Adenosine monophosphate-activated protein kinase (AMPK) is a highly conserved serine/threonine protein kinase consisting of a catalytic $\alpha$-subunit and regulatory $\beta$ - and $\gamma$-subunit. The $\alpha$-subunit contains a conserved threonine residue (Thr172) which was phosphorylated by upstream protein kinases leading to AMPK activation [20]. AMPK is a key cellular energy sensor activated by increasing AMP levels, coordinates cell growth with energy availability, and is also inactivated in an obese state. Cellular energy stress induces YAP phosphorylation, in part due to AMPKdependent Lats activation, thereby inhibiting YAP activity. In addition, AMPK directly phosphorylated YAP S94, a residue essential interacting with TEAD, thus disrupting the YAP-TEAD interaction [21]. Therefore, the AMPK-YAP pathway links cellular energy status such as obesity to tumorigenesis and may play a significant role in the growth and development of obesity-related cancers. EC is available for early lymphatic and haematogenous dissemination, and that is closely related to tumor invasion and metastasis [22]. Matrix metalloproteinases (MMPs) function as proteases for proteolytic cleavage of the extracellular matrix (ECM), necessary for tumor invasion and metastasis. MMP2 and MMP9, as important members of the gelatinase family of MMPs which degraded type IV collagen, the principal component of the basement membrane, cause increased tumor metastasis $[23,24]$. In particular, upregulated MMP9 is related to a poorer prognosis in a number of malignant tumors including ESCC and EAC [25-27].

Very little fundamental research has investigated the mechanisms by which obesity affects ESCC growth and development, and no suitable animal models about that exist currently. Therefore, an in vivo animal model and cell experiments in vitro were adopted in the study to explore the impact of obesity on ESCC growth and development and the related molecular mechanism.

\section{Materials and Methods}

2.1. Animals and Cell Culture. Animal experiments were approved by the Xi'an Jiaotong University Institutional Animal Care and Use Committee. Four- to five-week-old male nude mice obtained from Slac Laboratory animal (Shanghai, China) were housed in standard conditions, then divided into two groups which were fed a high-fat diet $(35.0 \%$ fat, $26.0 \%$ carbohydrate, and $26.0 \%$ protein) and normal chow (4.3\% fat, $67.3 \%$ carbohydrate, and $19.2 \%$ protein) [28] for 8 weeks, respectively. TE-1, kyse150, and Eca109 as typical ESCC cell lines were conferred from the experiment platform of Xi'an Jiaotong University or purchased from the cell bank of the Chinese Academy of Sciences, Shanghai, China. All cancer cells were cultured in a Roswell Park Memorial Institute-1640 (RPMI-1640) medium (Cellgro, Herndon, VA) and supplemented with $10 \%(v / v)$ heat-inactivated fetal bovine serum (Valley Biomedical, Winchester, VA), penicillin $(100 \mathrm{U} / \mathrm{mL})$, and streptomycin $(100 \mathrm{mg} / \mathrm{mL})$. Cells were maintained in a $37^{\circ} \mathrm{C}$ humidified incubator supplying $5 \%$ $\mathrm{CO}_{2}$. TE-1 was chosen for further trials depending on the protein expression of MMP9.

2.2. In Vivo ESCC Model. After 8 weeks, mice fed normal chow were defined as "lean," and mice consuming high-fat diet and collected by the criterion that the body weight exceeded the mean plus 2-fold standard deviation (SD) of these lean nude mice were defined as "obese," the animals left consuming the high-fat diet were referred to as "nonobese." All mice were inoculated subcutaneously with $2.0 \times 10^{6} \mathrm{TE}$ 1 cells into the right flank and monitored daily; then, those chosen nude mice were maintained on normal or high-fat diet for another 4 weeks, respectively. Tumor volume was assessed by gauging the length and width of the tumor with calipers as $1 / 2\left(\right.$ length $\times$ width $\left.^{2}\right)$, once tumors became palpable. After 4 weeks of injected tumor cell growth, all mice were sacrificed after anesthesia, and blood drawn from the retroorbital venous plexus was preserved for assessment of metabolites or adipokines. Tumors were carefully dissected, weighed, and then preserved for further analysis. Laparotomy was carried out to detect the existence of metastasis; then, the liver was resected and weighed and also preserved for oil red $\mathrm{O}$ staining.

2.3. Serum Assays. An enzyme-linked immunosorbent assay (ELISA) was utilized to determine the serum level of insulin, leptin, and visfatin following the manufacturer's instructions. 
TABLE 1: Sequences of the primers in the RT-PCR assay.

\begin{tabular}{lccc}
\hline Genes & Primer & $5^{\prime} \rightarrow 3^{\prime}$ sequences & Product size (bp) \\
\hline \multirow{2}{*}{ MMP9 } & Forward & CGCTGGGCTTAGATCATTCC & 107 \\
& Reverse & GTGCCGGATGCCATTCAC & 76 \\
AMPK & Forward & GTAGTAAAAACAGGCTCCACGAA & \\
& Reverse & CACCAGAAAGGATCTGTTGGA & 131 \\
YAP & Forward & CGCTCTTCAACGCCGTCA & 224 \\
& Reverse & AGTACTGGCCTGTCGGGAGT & 22 \\
GAPDH & Forward & ACTCCACGACGTACTCAGCG & \\
& Reverse & GGTCGGACTCAACGGATTTG & \\
\hline
\end{tabular}

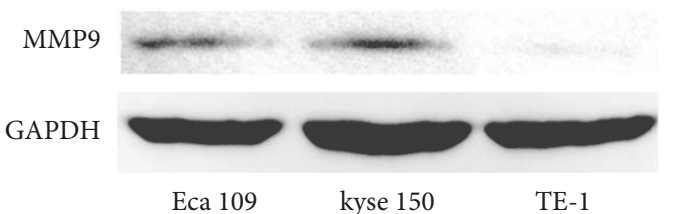

(a)

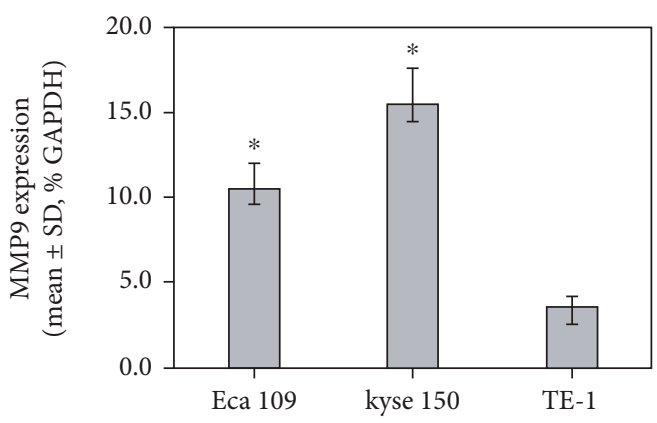

(b)

Figure 1: MMP9 protein expression in ESCC cell lines. ${ }^{*} P<0.05$ versus TE-1.

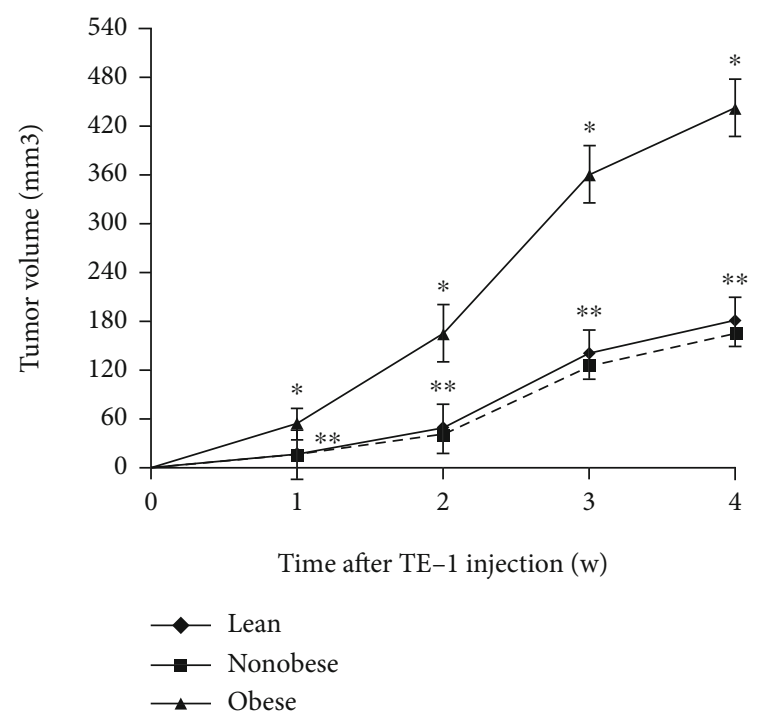

FIgURE 2: Subcutaneous tumor xenograft growth curve. Tumor xenografts from obese nude mice grew faster than those from lean and nonobese animals within 4 weeks. ${ }^{*} P<0.05$ versus lean and nonobese, ${ }^{* *} P>0.05$ versus nonobese.

All of the ELISA kits were purchased from Proteintech Group (Rosemont, USA). Serum glucose was assessed by a colorimetric assay.

2.4. Cellular Proliferation Assay. Cellular proliferation in vitro was estimated by the MTT assay. After exposure to
TABLE 2: The weight of body, liver, and their subcutaneous tumors was analyzed.

\begin{tabular}{lccc}
\hline Parameters & Obese & Lean & Nonobese \\
\hline Body weight $(\mathrm{g})$ & $30.18 \pm 0.67^{*}$ & $26.02 \pm 2.32^{* *}$ & $25.15 \pm 2.35$ \\
Liver weight $(\mathrm{g})$ & $1.03 \pm 0.09^{*}$ & $0.79 \pm 0.08^{* *}$ & $0.81 \pm 0.08$ \\
Tumor weight $(\mathrm{g})$ & $0.44 \pm 0.25^{*}$ & $0.18 \pm 0.15^{* *}$ & $0.16 \pm 0.14$ \\
\hline
\end{tabular}

${ }^{*} P<0.05$ versus lean and nonobese, ${ }^{* *} P>0.05$ versus nonobese.

conditioned media or RPMI-1640, TE-1 cells were incubated with $0.5 \%$ MTT solution for another $4 \mathrm{~h}$; then, $150 \mu \mathrm{L}$ of DMSO was introduced to solubilize the MTT tetrazolium crystal; optical density of them was determined at $490 \mathrm{~nm}$ by a Benchmark Plus microplate reader (Bio-Rad, Hercules, CA). All trials were repeated three times.

2.5. Migration and Invasion Assays. In order to determine the migration and invasion of TE-1 cells in response to exposure to conditioned media, migration and invasion assays were performed according to the instructions. Briefly, $5 \times 10^{4}$ TE- 1 cells per chamber were introduced to the top wells of migration chambers or invasion chambers with a matrigel membrane. Conditioned media or serum-free RPMI-1640 was placed into the wells beneath the chambers for $24 \mathrm{~h}$. After removing the cells from the upper surface of the filters, the remnant cells on the lower surface were counted randomly in 10 different high-power fields (magnification, $\times 200$ ) using a microscope. 


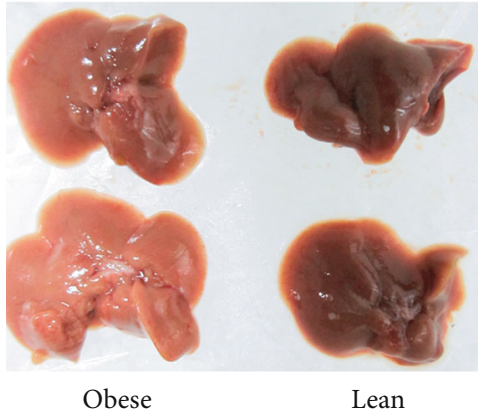

(a)

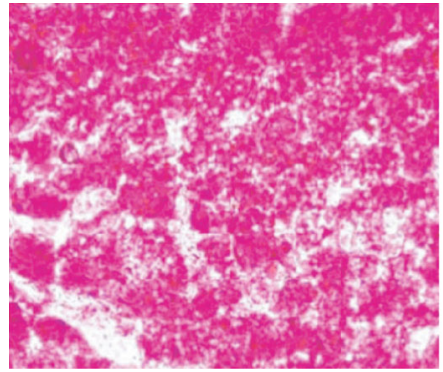

Obese

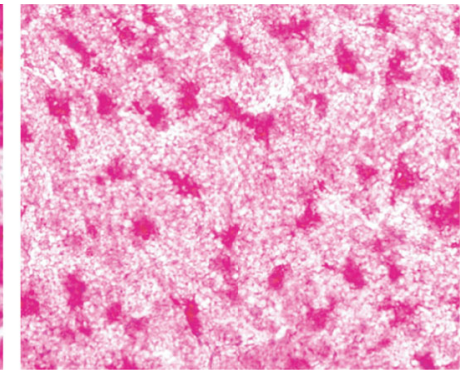

Lean

(b)

FIgURE 3: Liver character from obese and lean mice. (a) Unaided observation of the liver showed that the color of the liver became more yellow. (b) Oil red $\mathrm{O}$ staining of the liver specimen under a microscope demonstrated that lipid in cytoplasm became red with the help of oil red $\mathrm{O}$, and the fields of the liver specimen from the obese group get more red than that from lean mice. Magnification, $\times 400$.

2.6. RNA Expression Studies. Total RNA was extracted from target cells with exposure to conditioned media or RPMI1640 for $24 \mathrm{~h}$, with the help of the TRIzol reagent (Invitrogen, San Diego, CA), then reverse transcribed to cDNA with a cDNA Synthesis Kit (Takara Biochemicals, Japan) following the manufacturer's instructions. Expression of AMPK and YAP mRNA was quantified by RT-PCR (Takara Biochemicals, Japan). Transcript levels were normalized to GAPDH. Briefly, the reaction was performed utilizing an iCycler (Bio-Rad, Hercules, CA) with the following thermal profile: a preheating step at $95^{\circ} \mathrm{C}$ for $10 \mathrm{~min}, 30$ repeats of $94.0^{\circ} \mathrm{C}$ for $30 \mathrm{sec}, 57.0^{\circ} \mathrm{C}$ for $30 \mathrm{sec}$, and then $72^{\circ} \mathrm{C}$ for $1 \mathrm{~min}$. These primers' sequence was generated by NCBI Primer-BLAST (Table 1). For validation, each trial was done three times.

2.7. Western Blotting Analysis. Target cells exposed to conditioned media or RPMI-1640 for $24 \mathrm{~h}$ or target tumor tissues' extracts were analyzed by Western blotting as previously described to determine whether obesity may influence the growth and development of TE-1 [29]. Briefly, $25 \mu \mathrm{g}$ of extracted protein was resolved by SDS-PAGE and then electroblotted onto nitrocellulose membranes for Western blotting analysis. Blots were probed with recommended diluted primary antibodies overnight at $4{ }^{\circ} \mathrm{C}$, followed by incubation with HRP-conjugated secondary antibody for $1 \mathrm{~h}$ at room temperature. The membranes were developed with enhanced chemiluminescence (Pierce) by an enhanced chemiluminescence detection system (Amersham Bioscience, Piscataway, NJ, USA). The primary antibodies recognizing AMPK, pAMPK, YAP, p-YAP, and GAPDH were purchased from Beijing Biosynthesis Biotechnology (China).

2.8. Statistical Analysis. Values were expressed as the mean \pm SD. Statistical differences were evaluated by one-way analysis of variance (ANOVA) followed by Dunnett's test; correlation analysis was estimated by the Pearson test. Those $P<0.05$ were considered statistically significant.

\section{Results}

3.1. Expression of MMP9 Protein in ESCC Cell Lines. One of the key projects of this study was to investigate the invasion of ESCC, and MMP9 protein expression in common ESCC
TABLE 3: Serum metabolic and adipokine changes in mice.

\begin{tabular}{lccc}
\hline Parameters & \multicolumn{3}{c}{ Groups } \\
& Lean & Nonobese \\
\hline Insulin $(\mathrm{ng} / \mathrm{mL})$ & $0.51 \pm 0.05^{*}$ & $0.43 \pm 0.04$ & $0.42 \pm 0.05^{* *}$ \\
Glucose $(\mathrm{mmol} / \mathrm{L})$ & $11.4 \pm 0.77^{*}$ & $8.13 \pm 0.76$ & $7.65 \pm 0.84^{* *}$ \\
HOMA-IR & $5.49 \pm 0.61^{*}$ & $3.28 \pm 0.45$ & $3.02 \pm 0.52^{* *}$ \\
Leptin $(\mathrm{ng} / \mathrm{mL})$ & $0.25 \pm 0.04^{*}$ & $0.18 \pm 0.03$ & $0.17 \pm 0.02^{* *}$ \\
Visfatin $(\mathrm{ng} / \mathrm{mL})$ & $90.55 \pm 3.83^{*}$ & $73.22 \pm 10.54$ & $74.47 \pm 6.02^{* *}$ \\
\hline
\end{tabular}

${ }^{*} P<0.05$ versus lean and nonobese, ${ }^{* *} P>0.05$ versus lean.

cell lines such as Eca109, kyse150, and TE-1 was analyzed. The result of this study demonstrated that TE-1 had the lowest expression among them (Figure 1) and was chosen as the core cell line for further trials.

3.2. Metabolic Changes in Mice. Eight weeks later, 15 nude mice fed the normal chow were defined as "lean," 14 out of 40 nude mice consuming the high-fat diet were chosen as "obese," and then, the 26 nude mice left from the high-fat diet group were classified as "nonobese" mice. At the time of inoculating the animals, these obese mice were significantly heavier than lean animals $(28.04 \pm 0.53$ versus $23.84 \pm 1.86$ g, $P<0.001)$, but no difference was observed between lean and nonobese mice $(23.84 \pm 1.86$ versus $22.01 \pm 2.32 \mathrm{~g}, P=$ $0.42)$.

3.3. Tumor Growth and Development. Injected nude mice were fed the normal or high-fat diet for another 4 weeks. All of these mice were alive, and no metastasis was observed during the experimental time frame. Tumor growth was detected in $93.3 \%(14 / 15)$ of lean mice, in $92.9 \%(13 / 14)$ of obese mice, and in $88.5 \%(23 / 26)$ of nonobese animals. Tumors grew larger and faster in obese nude mice than lean and nonobese nude mice in the 4 weeks (Figure 2). By the end of the animal trial, the body, tumor, and liver weight of mice was collected (Table 2). Tumor weight indicated a strongly positive correlation with body weight of mice $\left(r_{s}=0.57, P\right.$ $<0.001)$. The obese mice also demonstrated a fatty liver (Figure 3), and tumor weight also revealed a strongly positive correlation with liver weight of mice $\left(r_{s}=0.62, P<0.001\right)$. 


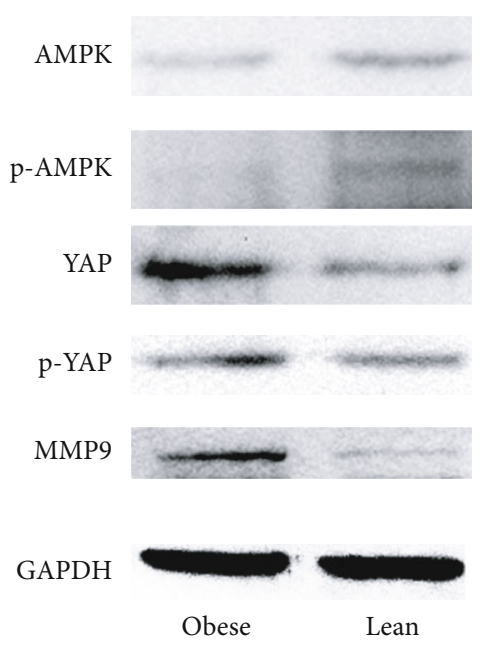

(a)

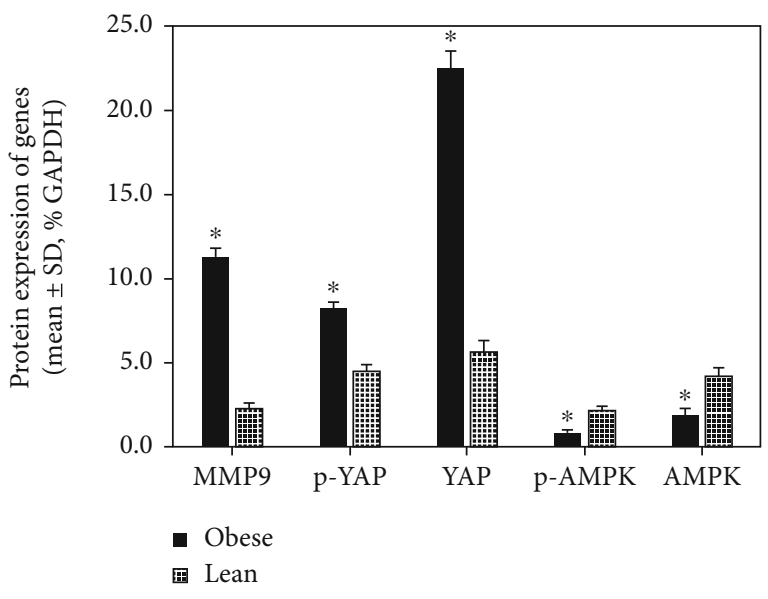

(b)

FIgure 4: MMP9, AMPK, and YAP protein expression in these subcutaneous tumors was evaluated semiqualitatively. ${ }^{*} P<0.05$ versus lean.

3.4. Metabolic Parameters and Adipokines. Glucose, insulin, leptin, and visfatin levels in sera, as well as the homeostatic model assessment insulin resistance (HOMA-IR) score, which is a measure of insulin resistance, were collected (Table 3). These obese nude mice demonstrated an altered metabolism milieu such as hyperglycemia, hyperinsulinemia, and insulin resistance and had a higher serum level of leptin and visfatin than lean mice by the end of the animal experiment. Tumor weight correlated positively with glucose $\left(r_{s}=0.50, P<0.001\right)$, HOMA-IR $\left(r_{s}=0.45, P=0.001\right)$, leptin $\left(r_{s}=0.34, P=0.017\right)$, and visfatin $\left(r_{s}=0.49, P<0.001\right)$ but is not correlated with insulin $\left(r_{s}=0.26, P=0.064\right)$.

3.5. MMP9, AMPK, and YAP Protein Expression in Tumor Tissues. Although no local and peritoneal metastasis was detected in the mice, related genes about tumor growth and development were still analyzed in the xenograft tumors by Western blotting. Tumors from obese nude mice had upregulated expression of MMP9, YAP, and p-YAP and lower expression of AMPK and p-AMPK than that from lean mice significantly (Figure 4). Further analysis showed that the active state of YAP, nonphosphorylated YAP, was higher in the tumor from the obese group than the lean group $(14.3 \pm 0.3$ versus $1.1 \pm 0.4 \% \mathrm{GAPDH}, P<0.001)$.

3.6. Effects of Obesity on Tumor Cell Proliferation, Migration, and Invasion. In order to detect the role of endocrine effect from obesity, cell experiments in vitro were performed with TE-1 cells under conditioned media. Conditioned media contained 5\% sera $(v / v)$ obtained from obese or lean mice and 95\% RPMI-1640. The MTT assay was used to assess TE-1 cell proliferation. These groups included obese, lean, and RPMI-1640 as the control group. After treatment, cell proliferation by the MTT assay was followed over a course of 5 days. The cell growth curve was made, and the results demonstrated that obesity prompted TE-1 cells to grow

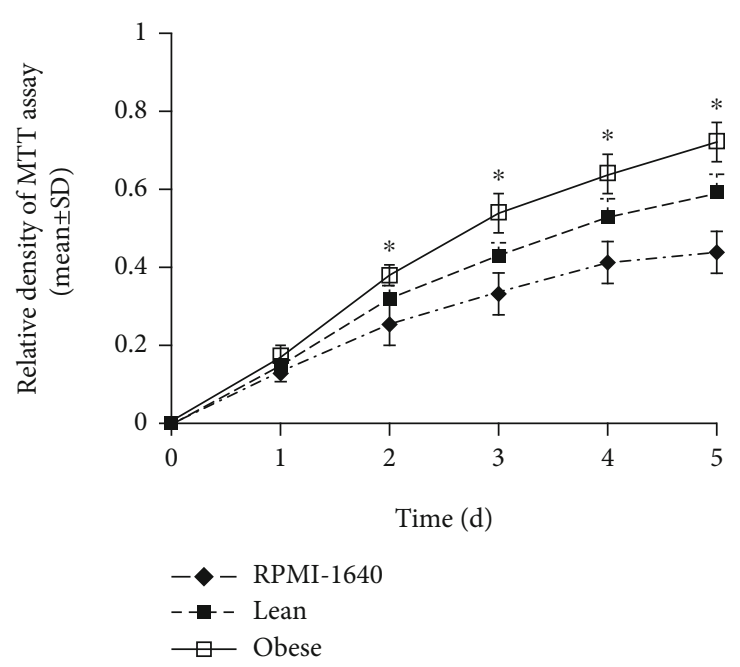

FIgURE 5: TE-1 cell proliferation in different states. TE-1 cultured with conditioned medium from sera of obese mice grew faster than those from lean or RPMI-1640. ${ }^{*} P<0.05$ vs. lean.

significantly faster compared to the lean group $(P<0.05$, Figure 5).

Individual cell migration and invasion is a significant feature of cancer cells to form local or distant metastasis, so a cell migration and invasion assay was performed. The migrating cell quantity of the obese group was significantly larger than that of the lean group in the high-power fields (magnification, $\times 200) \quad(270.1 \pm 19.8$ versus $158.0 \pm 21.6$ /field, $P<0.001$, Figure 6), and no cell migration existed in the RMPI-1640 group. The invasive cell quantity of the obese group was also significantly larger than that of the lean group in the high-power fields $(234.0 \pm 26.9$ versus $112.4 \pm 18.9$ /field, $P<0.001$, Figure 7). The results showed that obesity functioning as a chemokine induced TE-1 cells traversing 


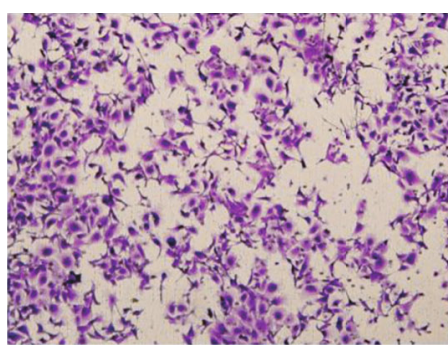

Obese

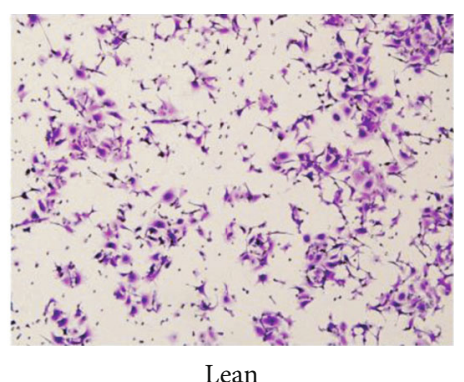

(a)

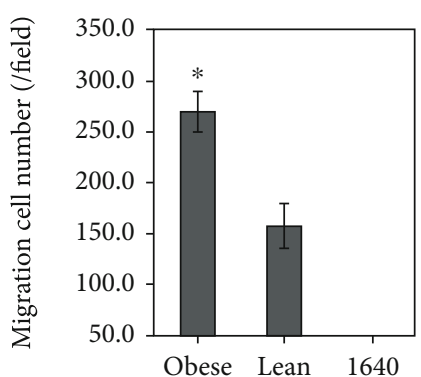

(b)

FIGURE 6: Effects of obesity on TE-1 cell migration. Obesity prompted significantly TE- 1 cell migration than the lean $(270.1 \pm 19.8$ versus $158.0 \pm 21.6 /$ field, $\left.{ }^{*} P<0.001\right)$. Magnification, $\times 200$.

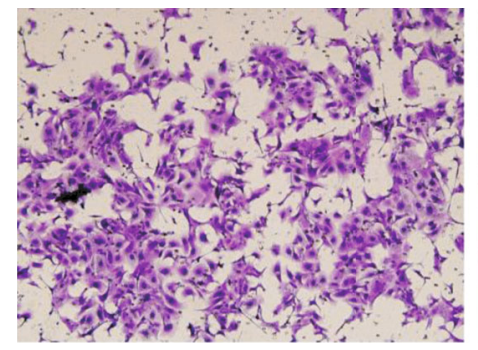

Obese

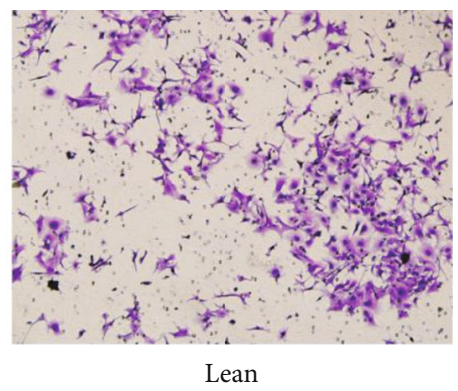

(a)

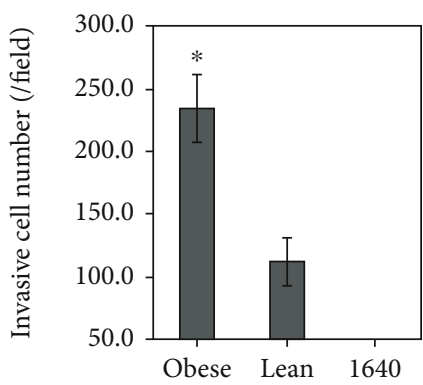

(b)

FIGURE 7: Effects of obesity on TE-1 cell invasion. Obesity accelerated significantly TE-1 cell invasion than the lean (234.0 \pm 26.9 versus $112.4 \pm 18.9 /$ field, $\left.{ }^{*} P<0.001\right)$. Magnification, $\times 200$.

the filter and prompted some MMP expression to act upon proteolytic cleavage of the matrigel membrane, then traverse it.

3.7. Obesity Promoting Growth and Development of ESCC by $M M P 9$ and $A M P K-Y A P$. For validation and further analysis of the endocrine mechanism from obesity, the expression of these related genes MMP9, AMPK, and YAP in the cultured TE-1 cells under different conditions was investigated. mRNA expression of AMPK decreased significantly $(P<0.05)$; YAP and MMP9 mRNA expression elevated significantly $(P<0.05)$ in the cells cultured with conditioned media from the obese group. Protein expression of AMPK and p-AMPK declined significantly $(P<0.05)$; MMP9, total YAP, and p-YAP protein was significantly upregulated $(P<0.05)$ in TE-1 cells in the context of obesity (Figure 8$)$. Further analysis also revealed that nonphosphorylated YAP expression still rose in TE-1 cells cultured with conditioned media from the obese group than that from the lean group (33.4 \pm 0.5 versus $20.0 \pm 0.9 \% \mathrm{GAPDH}, P<0.001)$. Therefore, obesity could potentiate TE- 1 cell proliferation, migration, and invasion and altered gene expression by endocrine effect.

\section{Discussion}

Obesity confers increased risk for a variety of serious conditions and is increasingly recognized as a cause of preventable cancer risk such as ESCC. The mechanisms linking obesity with ESCC are complicated since lots of biological effects of obesity exist, such as tumor microenvironment, abnormal adipokine secretion, oxidative stress, metabolites, inflammation, immunity, and complex effect [12]. These effects can be categorized into cancer-stromal interaction, paracrine, autocrine, and endocrine mechanism [30]. Altered metabolic activity of visceral AT in obesity may play a fundamental role in the development of these obesity-associated cancers, and AT may influence the progression of ESCC by increased cancer cell growth or invasion and decreased apoptosis [31]. Cancer-stromal interaction driven by cell-cell contact and autocrine, paracrine, and endocrine mechanisms is critical for the growth, invasion, and metastasis of various cancers such as EC [32].

Therefore, we developed modeling ESCC in vivo and cell trials in vitro utilizing the sera of mice to determine this correlation between obesity and ESCC in the study. The results demonstrated that TE-1 xenograft tumors grew larger in obese nude mice than that in lean animals, which strongly demonstrated the positive correlation between obesity and ESCC growth, providing powerful statistic support for the direct effect of obesity on ESCC growth. The results also showed that obesity could potentiate TE- 1 cell proliferation, migration, and invasion by the endocrine mechanism. Obesity increased the expression of proproliferative and antiapoptotic genes, which contributed to the survival of TE-1. This study was a novel in vivo animal model of ESCC growth 

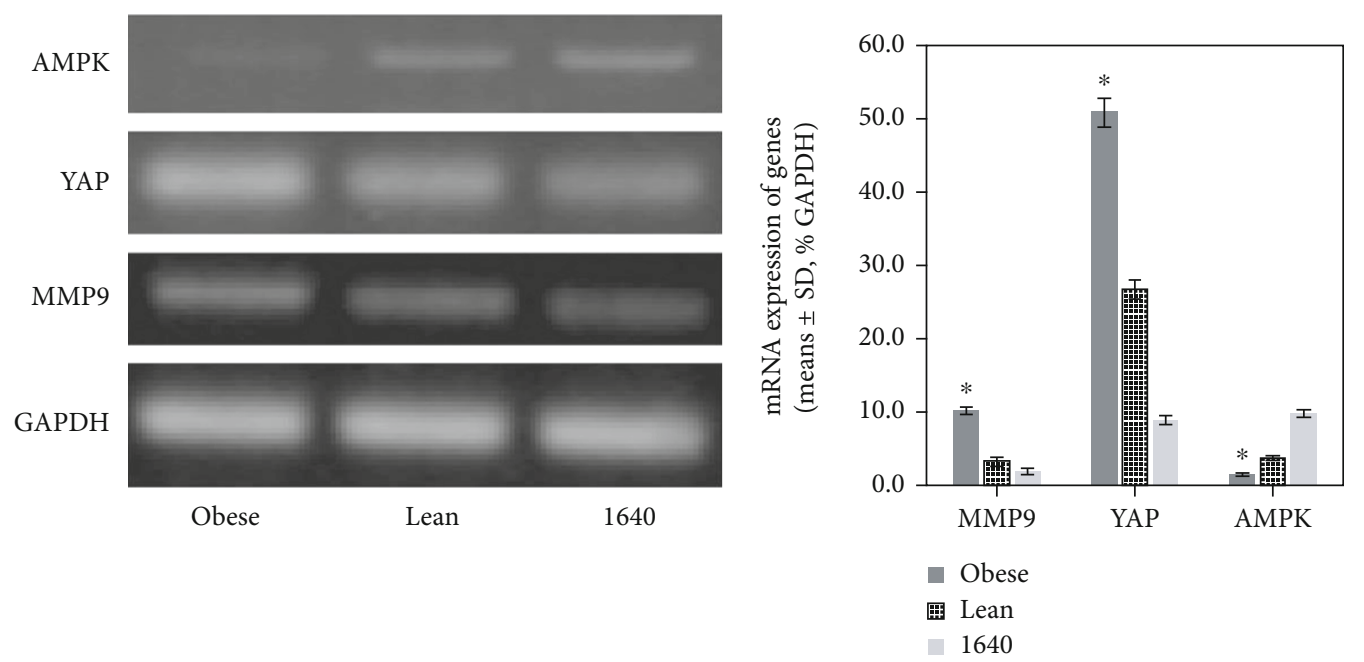

(a)
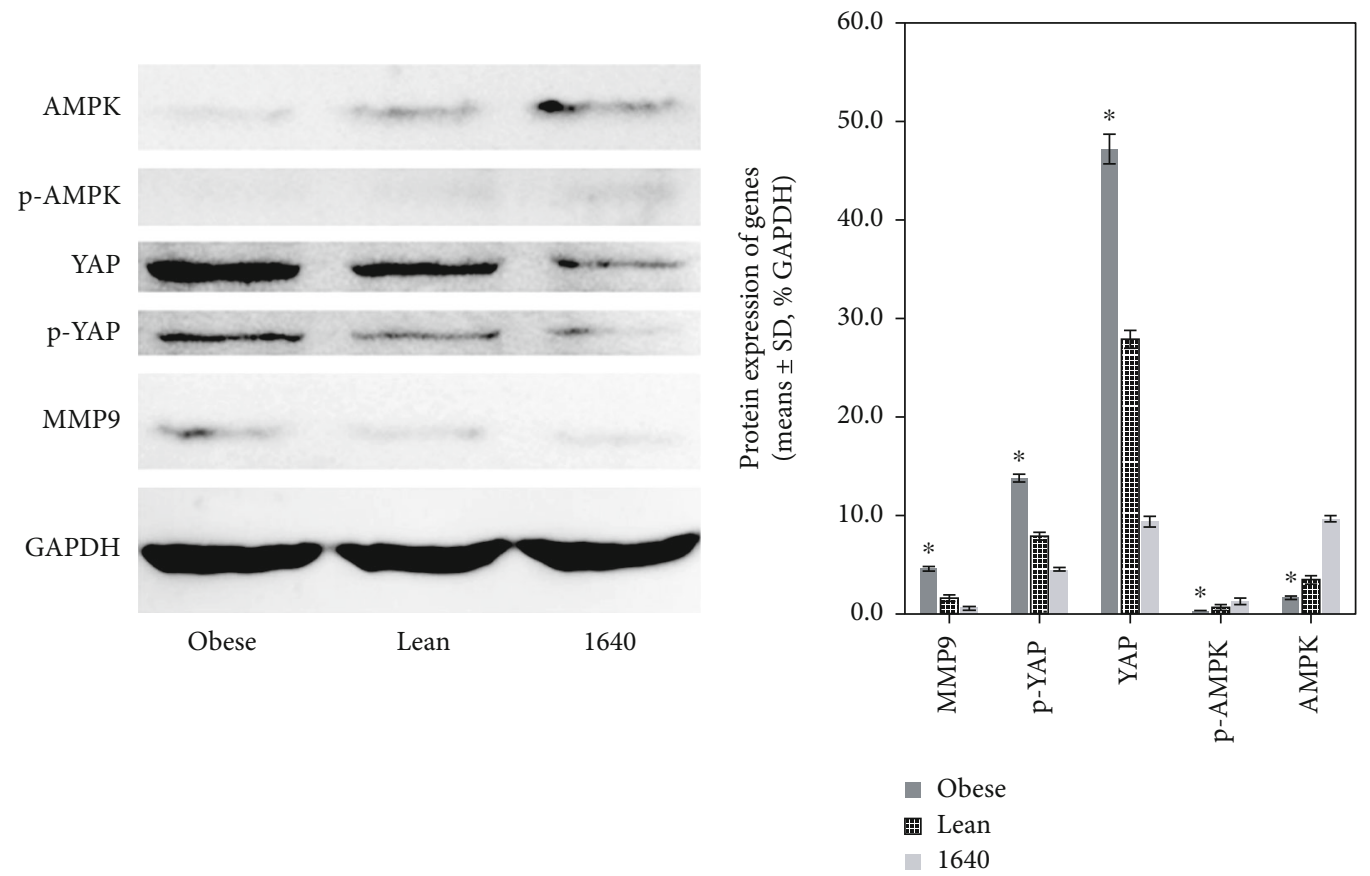

(c)

(d)

FIGURE 8: Related mechanism that diet-induced obesity accelerated TE-1 cell growth and progression was dissected. Obesity prompted the growth and development of TE-1 cells by MMP9 and AMPK-YAP signaling pathway at the mRNA level by RT-PCR (a, b) and at the protein level by Western blotting (c, d). ${ }^{*} P<0.05$ versus lean.

under the background of obesity by nude mice. Though nude mice are not prone to obesity, they are good candidates for the xenograft tumor, which make them suitable subjects for this study, and up to $35 \%$ (14/40) mice were chosen by the criterion as obese mice for further trials in the study. The model could also be used to further explore the mechanisms of how obesity affects ESCC growth. Tumorigenesis in obese patients is determined by a series of important mechanisms and metabolites such as insulin, insulin resistance, leptin, and visfatin $[33,34]$, while a strong positive correlation was observed between glucose, HOMA-IR, leptin, and visfatin in sera and xenograft tumor growth in this study. Despite these findings, it remains to be examined which specific adipose depots or obese effects are relevant to cancer growth and development.

Adipokines such as leptin and adiponectin are implicated in cell growth, proliferation, cell cycle control, and angiogenesis [12]; the results of this study also verified the relationship between ESCC growth and leptin. Among adipokines, growing interest has been placed in recent years on visfatin and its role in carcinogenesis. Visfatin may prompt tumor proliferation and metastasis in a number of cancers such as breast cancer [35], oral squamous cell carcinoma [36], and gastric cancer [37]. This study also proved the relationship between ESCC growth and visfatin. Visfatin is also functioning as a NAD biosynthetic enzyme which was overexpressed in 
multiple cancers including gastric cancer [38] and oral squamous cell carcinoma [39]. Another prominent adipokine, adiponectin, is mainly secreted from visceral AT but negatively related to adiposity, hyperinsulinemia, and inflammation. Adiponectin may exert anticancer effects by activation of AMPK [40]. AMPK is an important cellular energy sensor which is activated by increasing AMP levels. It works by coordinating cell growth with energy availability and is also inactivated in an obese state. Hence, the AMPK-YAP signaling pathway is closely related to the cellular energy state including glucose and lipid metabolism such as obesity and cancer. This study is the first to prove that obesity potentiated ESCC growth and invasion with relation to the AMPK-YAP signal pathway and reveal that obesity downregulated AMPK and p-AMPK, while upregulating MMP9, total YAP, p-YAP, and nonphosphorylated YAP protein expression. It is also found that obesity reduced AMPK and enhanced YAP and MMP9 expression in the mRNA level by complex effect including an important pathway, the endocrine mechanism.

IR was related to increased lung cancer risk [41], and insulin may also influence obesity-mediated tumors such as renal cancer and ovarian tumor $[42,43]$, but the relationship between insulin and ESCC growth had not been examined in the study. The novel findings of this study that serum visfatin and leptin were positively related to xenograft tumor growth were consistent with the notion that adipokines and other growth factors secreted on the background of obesity may prompt cancer cell survival and tumor growth. Certainly, limitations of the study existed; one of them is that nude mice are insensitive to establishing an obese model, and nude mice have only a small volume of serum unavailable for further analysis such as adiponectin and lipid assay. Another is that the complicated effect of obesity on the survival of mice bearing tumors could not be estimated.

\section{Conclusion}

IR and changed adipokine milieu observed in obesity may potentiate ESCC xenograft tumor growth and promote ESCC cell proliferation, migration, and invasion in vitro.

\section{Data Availability}

The raw data of correlation analysis between serum glucose, insulin, HOMA-IR, leptin, visfatin, body weight, and liver weight of mice and weight of xenograft tumors which was not shown in the manuscript is available from the corresponding authors by request. All of the other data is provided within the manuscript.

\section{Conflicts of Interest}

The authors declare that they have no conflict of interests.

\section{Authors' Contributions}

Xiang-Ming Che and Hai-Jun Li contributed equally to this work.

\section{Acknowledgments}

This study was supported by a grant from the National Natural Science Foundation of China (No.81703079).

\section{References}

[1] L. A. Torre, F. Bray, R. L. Siegel, J. Ferlay, J. Lortet-Tieulent, and A. Jemal, "Global cancer statistics, 2012," CA: A Cancer Journal for Clinicians, vol. 65, no. 2, pp. 87-108, 2015.

[2] W. Chen, R. Zheng, P. D. Baade et al., "Cancer statistics in China, 2015," CA: A Cancer Journal for Clinicians, vol. 66, no. 2, pp. 115-132, 2016.

[3] F. L. Huang and S. J. Yu, "Esophageal cancer: risk factors, genetic association, and treatment," Asian Journal of Surgery, vol. 41, no. 3, pp. 210-215, 2018.

[4] The GBD 2015 Obesity Collaborators, "Health effects of overweight and obesity in 195 countries over 25 years," The New England Journal of Medicine, vol. 377, no. 1, pp. 13-27, 2017.

[5] J. Tian, C. Zuo, G. Liu et al., "Cumulative evidence for the relationship between body mass index and the risk of esophageal cancer: an updated meta-analysis with evidence from 25 observational studies," Journal of Gastroenterology and Hepatology, vol. 35, no. 5, pp. 730-743, 2020.

[6] K. I. Avgerinos, N. Spyrou, C. S. Mantzoros, and M. Dalamaga, "Obesity and cancer risk: emerging biological mechanisms and perspectives," Metabolism, vol. 92, pp. 121-135, 2019.

[7] G. A. Colditz and L. L. Peterson, "Obesity and cancer: evidence, impact, and future directions," Clinical Chemistry, vol. 64, no. 1, pp. 154-162, 2018.

[8] I. Trestini, L. Carbognin, C. Bonaiuto, G. Tortora, and E. Bria, "The obesity paradox in cancer: clinical insights and perspectives," Eating and Weight Disorders - Studies on Anorexia, Bulimia and Obesity, vol. 23, no. 2, pp. 185-193, 2018.

[9] J. H. Cho, C. M. Shin, K. D. Han et al., "Abdominal obesity increases risk for esophageal cancer: a nationwide population-based cohort study of South Korea," Journal of Gastroenterology, vol. 55, no. 3, pp. 307-316, 2020.

[10] A. Okamura, M. Watanabe, S. Mine et al., "Clinical impact of abdominal fat distribution on prognosis after esophagectomy for esophageal squamous cell carcinoma," Annals of Surgical Oncology, vol. 23, no. 4, pp. 1387-1394, 2016.

[11] H. Tilg and A. R. Moschen, "Adipocytokines: mediators linking adipose tissue, inflammation and immunity," Nature Reviews Immunology, vol. 6, no. 10, pp. 772-783, 2006.

[12] T. Deng, C. J. Lyon, S. Bergin, M. A. Caligiuri, and W. A. Hsueh, "Obesity, inflammation, and cancer," Annual Review of Pathology, vol. 11, no. 1, pp. 421-449, 2016.

[13] J. E. Drew, "Molecular mechanisms linking adipokines to obesity-related colon cancer: focus on leptin," The Proceedings of the Nutrition Society, vol. 71, no. 1, pp. 175-180, 2012.

[14] A. Fukuhara, M. Matsuda, M. Nishizawa et al., "Visfatin: a protein secreted by visceral fat that mimics the effects of insulin," Science, vol. 307, no. 5708, pp. 426-430, 2005.

[15] A. A. Grolla, C. Travelli, A. A. Genazzani, and J. K. Sethi, "Extracellular nicotinamide phosphoribosyltransferase, a new cancer metabokine," British Journal of Pharmacology, vol. 173, no. 14, pp. 2182-2194, 2016.

[16] S. Ono, K. Nakano, K. Takabatake, H. Kawai, and H. Nagatsuka, "Immunohistochemistry of YAP and dNp63 and survival analysis of patients bearing precancerous lesion 
and oral squamous cell carcinoma," International Journal of Medical Sciences, vol. 16, no. 5, pp. 766-773, 2019.

[17] P. Li, D. Sun, X. Li et al., "Elevated expression of Nodal and YAP1 is associated with poor prognosis of gastric adenocarcinoma," Journal of Cancer Research and Clinical Oncology, vol. 142, no. 8, pp. 1765-1773, 2016.

[18] M. K. Yeo, S. H. Kim, J. M. Kim et al., "Correlation of expression of phosphorylated and non-phosphorylated Yesassociated protein with clinicopathological parameters in esophageal squamous cell carcinoma in a Korean population," Anticancer Research, vol. 32, no. 9, pp. 3835-3840, 2012.

[19] T. Moroishi, C. G. Hansen, and K. L. Guan, "The emerging roles of YAP and TAZ in cancer," Nature Reviews Cancer, vol. 15, no. 2, pp. 73-79, 2015.

[20] D. G. Hardie, "AMP-activated/SNF1 protein kinases: conserved guardians of cellular energy," Nature Reviews Molecular Cell Biology, vol. 8, no. 10, pp. 774-785, 2007.

[21] J. S. Mo, Z. Meng, Y. C. Kim et al., "Cellular energy stress induces AMPK-mediated regulation of YAP and the Hippo pathway," Nature Cell Biology, vol. 17, no. 4, pp. 500-510, 2015.

[22] S. M. Lagarde, F. J. W. ten Kate, D. J. Richel, G. J. A. Offerhaus, and J. J. B. van Lanschot, "Molecular prognostic factors in adenocarcinoma of the esophagus and gastroesophageal junction," Annals of Surgical Oncology, vol. 14, no. 2, pp. 977991, 2007.

[23] T. Turpeenniemi-Hujanen, "Gelatinases (MMP-2 and -9) and their natural inhibitors as prognostic indicators in solid cancers," Biochimie, vol. 87, no. 3-4, pp. 287-297, 2005.

[24] R. Zeng, J.-P. Huang, X. F. Li et al., "Epb4113 suppresses esophageal squamous cell carcinoma invasion and inhibits MMP2 and MMP9 expression," Cell Biochemistry and Function, vol. 34, no. 3, pp. 133-141, 2016.

[25] Z. D. Gu, J. Y. Li, M. Li et al., "Matrix metalloproteinases expression correlates with survival in patients with esophageal squamous cell carcinoma," The American Journal of Gastroenterology, vol. 100, no. 8, pp. 1835-1843, 2005.

[26] J. M. Hu, K. Liu, J. H. Liu et al., "CD163 as a marker of M2 macrophage, contribute to predicte aggressiveness and prognosis of Kazakh esophageal squamous cell carcinoma," Oncotarget, vol. 8, no. 13, pp. 21526-21538, 2017.

[27] E. H. Allott, J. Lysaght, M. C. Cathcart et al., "MMP9 expression in oesophageal adenocarcinoma is upregulated with visceral obesity and is associated with poor tumour differentiation," Molecular Carcinogenesis, vol. 52, no. 2, pp. 144-154, 2013.

[28] S. Yakar, N. P. Nunez, P. Pennisi et al., "Increased tumor growth in mice with diet-induced obesity: impact of ovarian hormones," Endocrinology, vol. 147, no. 12, pp. 5826-5834, 2006.

[29] H. Li, E. Bai, Y. Zhang, Z. Jia, S. He, and J. Fu, "Role of Nampt and visceral adiposity in esophagogastric junction adenocarcinoma," Journal of Immunology Research, vol. 2017, Article ID 3970605, 7 pages, 2017.

[30] K. M. Nieman, I. L. Romero, B. Van Houten, and E. Lengyel, "Adipose tissue and adipocytes support tumorigenesis and metastasis," Biochimica et Biophysica Acta (BBA) - Molecular and Cell Biology of Lipids, vol. 1831, no. 10, pp. 1533-1541, 2013.

[31] A. Nakayama, S. Aoki, K. Uchihashi et al., "Interaction between esophageal squamous cell carcinoma and adipose tis- sue in vitro," The American Journal of Pathology, vol. 186, no. 5, pp. 1180-1194, 2016.

[32] L. Fu, C. Zhang, L. Y. Zhang et al., "Wnt2 secreted by tumour fibroblasts promotes tumour progression in oesophageal cancer by activation of the $\mathrm{Wnt} / \beta$-catenin signalling pathway," Gut, vol. 60, no. 12, pp. 1635-1643, 2011.

[33] S. E. Ackerman, O. A. Blackburn, F. Marchildon, and P. Cohen, "Insights into the link between obesity and cancer," Current Obesity Reports, vol. 6, no. 2, pp. 195-203, 2017.

[34] S. Djiogue, A. H. Nwabo Kamdje, L. Vecchio et al., "Insulin resistance and cancer: the role of insulin and IGFs," Endocrine-Related Cancer, vol. 20, no. 1, pp. R1-R17, 2013.

[35] S.-H. Moi, Y.-C. Lee, L.-Y. Chuang et al., "Cumulative receiver operating characteristics for analyzing interaction between tissue visfatin and clinicopathologic factors in breast cancer progression," Cancer Cell International, vol. 18, no. 1, p. 19, 2018.

[36] T. Yu-Duan, W. Chao-Ping, C. Chih-Yu et al., "Elevated plasma level of visfatin/pre-b cell colony-enhancing factor in male oral squamous cell carcinoma patients," Medicina Oral Patología Oral y Cirugia Bucal, vol. 18, no. 2, pp. e180-e186, 2013.

[37] G. W. Lu, Q. J. Wang, M. M. Xia, and J. Qian, “Elevated plasma visfatin levels correlate with poor prognosis of gastric cancer patients," Peptides, vol. 58, pp. 60-64, 2014.

[38] T. Q. Bi, X. M. Che, X. H. Liao et al., "Overexpression of Nampt in gastric cancer and chemopotentiating effects of the Nampt inhibitor FK866 in combination with fluorouracil," Oncology Reports, vol. 26, no. 5, pp. 1251-1257, 2011.

[39] X. Y. Wang, J. Z. Wang, L. Gao et al., "Inhibition of nicotinamide phosphoribosyltransferase and depletion of nicotinamide adenine dinucleotide contribute to arsenic trioxide suppression of oral squamous cell carcinoma," Toxicology and Applied Pharmacology, vol. 331, pp. 54-61, 2017.

[40] A. Tumminia, F. Vinciguerra, M. Parisi et al., "Adipose tissue, obesity and adiponectin: role in endocrine cancer risk," International Journal of Molecular Sciences, vol. 20, no. 12, p. 2863, 2019.

[41] I. Argirion, S. J. Weinstein, S. Mannisto, D. Albanes, and A. M. Mondul, "Serum insulin, glucose, indices of insulin resistance, and risk of lung cancer," Cancer Epidemiology Biomarkers \& Prevention, vol. 26, no. 10, pp. 1519-1524, 2017.

[42] W. Solarek, A. M. Czarnecka, B. Escudier, Z. F. Bielecka, F. Lian, and C. Szczylik, "Insulin and IGFs in renal cancer risk and progression," Endocrine-Related Cancer, vol. 22, no. 5, pp. R253-R264, 2015.

[43] L. D. Kellenberger and J. Petrik, "Hyperglycemia promotes insulin-independent ovarian tumor growth," Gynecologic Oncology, vol. 149, no. 2, pp. 361-370, 2018. 